\title{
Study of Morbidity and Mortality Pattern of Children Admitted in Paediatric Intensive Care Unit of Tertiary Care Children's Hospital
}

\section{Prakash Joshi, Sumit Agrawal and Umesh Prasad Sah}

Department of Paediatrics, Kanti Children's Hospital, Maharajgunj, Kathmandu, Nepal

\author{
Correspondence: \\ Prakash Joshi \\ Department of Paediatrics, \\ Kanti Children's Hospital, Maharajgunj, \\ Kathmandu, Nepal \\ Email: dr.prakash346088@gmail.com
}

DOI: $10.3126 /$ jnps.v40i3.29108

Submitted on: $2020-05-25$

Accepted on: 2020-07-03

Acknowledgements: Nursing staffs of PICU and staffs of record section for preserving and providing the required data.

Funding: Nil

Conflict of Interest: None declared

Permission from IRB: Yes

To cite this article: Joshi $\mathrm{P}$, Agrawal S, Sah UP. Study of morbidity and mortality pattern of children admitted in paediatric intensive care unit of tertiary care children's hospital. J Nepal Paediatr Soc. 2020;40(3):232-40.

\begin{abstract}
Introduction: Intensive care is predominantly concerned with the management of patients with acute life threatening conditions in a specialised unit. The objective of this study was to describe the morbidity and mortality patterns as well as outcome of patients admitted in the Paediatric Intensive Care Unit (PICU) of a tertiary care government paediatric hospital in Nepal.
\end{abstract}

Methods: A cross-sectional study of 652 children admitted to the PICU of Kanti Children's Hospital was undertaken from $1^{\text {st }}$ January 2018 to $31^{\text {st }}$ December 2018. The data were retrieved retrospectively from the record files of PICU regarding patient's age, sex, inhabitant, admitting and final diagnosis, length of stay in the ICU and final outcome. Data were entered into MS Excel and analysed using Pearson's Chi Square Test.

Results: Among 652 admitted children between 1 month to 14 years of age, $397(61 \%)$ were males and $255(39 \%)$ females with male to female ratio $1.55: 1$. Majority $352(54 \%)$ of them were in the age group of one month to 12 months of age. Pneumonia (216, $33.1 \%)$ was the major disease seen followed by septicemia $(123$, $18.9 \%)$, bronchiolitis $(44,6.7 \%)$, other infectious diseases $(38$, $5.8 \%)$ and so on. Out of total admission, $484(74.23 \%)$ cases improved, 46 (7.05\%) left against medical advice (LAMA) and 120 cases died with mortality rate of $18.46 \%$. More than half of the deaths ( $\mathrm{n}=87 / 120,72.5 \%)$ were due to infections (Septicemia, acute gastroenteritis, pneumonia and meningitis and other infectious diseases).

Conclusions: Pneumonia with respiratory distress was the major cause of PICU admission while fatality was highest for acute leukemia. Therefore it seems justifiable to improve primary and secondary health care facilities for timely and reliable delivery of current standardised therapeutic practice as well as to increase ICU facilities in those areas.

Keywords: Admission pattern; Outcome; Paediatric Intensive Care; Survivor

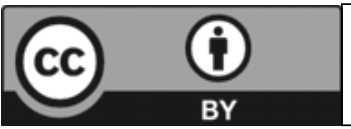

This work is licensed under creative common attribution 3.0 license 


\section{INTRODUCTION}

The care of critically ill children remains one of the most demanding and challenging aspects in the field of paediatrics. Paediatric intensive care unit (PICU) aims at promoting early intervention and quality care with an objective of achieving good results and better prognosis. ${ }^{1}$ Advances in knowledge and technology of medical science has dramatically improved the prognosis of critically-ill children. Numerous conditions that were previously fatal are now treatable. ${ }^{2}$

Though the specialty of paediatric critical care medicine has grown and matured over years in the developed countries, the concept of paediatric critical care is relatively new in Nepal. ${ }^{3}$ A growing demand for critical care services has led some of the major teaching hospitals to provide spaces for management of severely ill children, in spite of a lack of trained manpower, adequate infrastructures and appropriate knowledge. ${ }^{4}$ Paediatric intensive care admissions vary among different regions in different settings. The profile of children admitted mainly differs from western countries in terms of the demography, spectrum of diseases, incidence and outcomes. ${ }^{5}$ Respiratory, neurological and infectious diseases are more common in this part of the globe whereas solid tumours, trauma and neurological infections are more common in western world. ${ }^{6}$ There are several reports on the outcome of PICUs from developed countries, but only few reports are available from Nepal., $3,4,7$ Therefore we planned to carry out this study at the only central level Government's paediatric hospital with largest critical care unit catering services to the needy children referred from all over the country.

The main objective of the study was to determine the pattern and prevalence of morbidity, temporal distribution, geographical distribution, duration of hospital stay, outcome and mortality pattern among children between one month to 14 years of age admitted in PICU. Results thus obtained would be useful in modifying healthcare strategies if necessary, appropriate resource allocation and formulating appropriate interventions leading to better management and outcome of critically ill children.

\section{METHODS}

This cross sectional study was conducted in the PICU of Kanti Children's Hospital $(\mathrm{KCH})$, the largest tertiary care Government paediatric hospital in Nepal. Being the only tertiary care Government paediatric hospital in the country, the twelve bedded PICU with two extra isolation beds operates at full capacity all the time.

Record files of all the paediatric patients, aged one month to 14 years, admitted in PICU of $\mathrm{KCH}$ over a period of one year $\left(1^{\text {st }}\right.$ January 2018 to $31^{\text {st }}$ December 2018) were reviewed retrospectively out of which 652 admitted children's record were found to have complete data set and hence were included for analysis. Variables recorded were patient's age, gender, date of entry into and exit from PICU, duration of stay, diagnosis at admission and exit from PICU as well as final outcome. Diagnosis was classified as per international classification of disease. "Other Infectious Diseases" included tropical diseases like, tuberculosis, enteric fever, scrub typhus, pertusis like illness, chicken pox, kala-azar, tetanus, brucellosis and Croup. Address of the patient was categorised into district and provinces. Data were entered into MS Excel and exported to SPSS version 20.0 for analysis. Descriptive statistics were computed for all study variables with mean standard deviation and median used as measures of central tendency. Comparisons of proportions were performed using Pearson chisquare test with a $\mathrm{p}$ value of $<0.05$ considered as significant. The odds of a particular disease was calculated with respect to remaining all other diseases. The study was conducted after taking an Ethical approval from Intuitional Review Committee of Kanti Children's Hospital with ref no: 339/075-076. Formula used for calculating hospital statistics are as follows..$^{8,9}$

Length of stay $=$ Total inpatient - days stay $/$ Total Number of discharges and deaths

Mean cases per month $=$ Total number of cases admitted during study period / 12

Bed occupancy rate $=$ Number of inpatients-days $x$ 100 / No of inpatients-bed available x 365 
Table 1. Demographic Distribution of Patients

\begin{tabular}{|lrrr}
\hline \multirow{2}{*}{ Age group } & \multicolumn{2}{c}{ Gender } & \multicolumn{1}{c}{ Total(\%) } \\
\cline { 2 - 3 } & Male & Female & \\
\hline < 1 year & 214 & 138 & $352(54 \%)$ \\
1 - 4 years & 79 & 53 & $132(20.2 \%)$ \\
5 - 9 years & 69 & 37 & $106(16.3 \%)$ \\
$>$ 10 years & 35 & 27 & $62(9.5 \%)$ \\
Total & 397 & 255 & $652(100 \%)$ \\
\hline
\end{tabular}

Bed turn-over rate $=$ Number of discharges (including deaths) in a given time period / Number of beds available during that time period.

Case fatality rate $=$ Number of deaths by particular disease x 100 / Number of cases of that particular disease registered in the same year.

\section{RESULTS}

During one year of study period, a total of 652 patients were admitted in PICU (mean 54.3 cases/ month). The bed occupancy rate was $85.18 \%$ per year with the turnover rate of 50.33. Among the study population, 397 (61\%) were males and 255 (39\%) females, with a M:F ratio of 1.55:1. Age on admission ranged from one month to 14 years with a mean of $34.18 \pm 45.48$ months and median of nine months. Table 1 shows demographic distribution of the study population.

Pneumonia (33.1\%) was the major disease seen in our PICU followed by sepsis (18.9\%) and bronchiolitis $(6.7 \%)$. Various diseases encountered in PICU are as listed in Table 2. Diseases like bronchial asthma, rheumatic heart disease (RHD), PSGN, Bartter syndrome, renal tubular acidosis, SLE, liver cirrhosis, anaemia with complications, diabetes mellitus and complications etc were included in miscellaneous group.

System wise evaluation showed respiratory system $(\mathrm{n}=178,27.3 \%)$ and infectious group $(\mathrm{n}=157$, $24.1 \%$ ) at the top of the list followed by neurological cases $(\mathrm{n}=68,10.4 \%)$. Around 67 $(10.3 \%)$ cases had involvement of more than two systems. Figure 1 shows system wise distribution of cases in PICU. Almost one fifth $(\mathrm{n}=141$,
Table 2. Common Diseases in PICU

\begin{tabular}{llrr} 
S.N. & Name of Diseases & $\begin{array}{l}\text { No of } \\
\text { Patients }\end{array}$ & Percent \\
\hline 1 & Pneumonia & 216 & 33.1 \\
2 & Sepsis & 123 & 18.9 \\
3 & Bronchiolitis & 44 & 6.7 \\
4 & Other infectious diseases & 38 & 5.8 \\
5 & Seizure disorder & 24 & 3.7 \\
6 & AES & 23 & 3.5 \\
7 & AGE with severe & 19 & 2.9 \\
& dehydration & & \\
8 & Meningitis & 18 & 2.8 \\
9 & Congenital heart diseases & 17 & 2.6 \\
10 & Acute leukemia & 16 & 2.5 \\
11 & Other oncological & 14 & 2.1 \\
& disorders & & \\
12 & Nephrotic syndrome & 12 & 1.8 \\
13 & Poisoning & 11 & 1.7 \\
14 & GBS & 6 & 0.9 \\
15 & Miscellaneous & 71 & 10.88 \\
16 & Total & 652 & 100 \\
\hline AES: Acute Enchephalitis Syndrome & & \\
AGE: Acute Gastroenteritis & & \\
GBS: Guillain-Barré syndrome & &
\end{tabular}

$21.62 \%)$ of the cases were having underlying comorbid conditions mainly congenital heart diseases (CHD) $(\mathrm{n}=68)$, Down's phenotype with complications $(n=23)$ and global developmental delay (GDD) ( $n=13)$. Besides, cerebral palsy (CP), failure to thrive (FTT), protein energy malnutrition (PEM), acute Leukemia and paediatric surgical problems like $\mathrm{CDH}, \mathrm{HD}$, gastroschiasis, etc were less common co-morbid association. Around 13 cases were found to be readmitted during the study period.

The main reason for PICU admission was respiratory distress including ARDS $(\mathrm{n}=401$, $61.50 \%$ ) with underlying respiratory, cardiac and metabolic diseases. Around $40(6.13 \%)$ patients were admitted because of shock, majority of which was septic shock 28 (4.29\%). Other causes included status epilepticus with altered mental status and poor GCS, $35(5.36 \%)$ mainly with the diagnoses of meningitis, meningoencephalitis and hepatic 


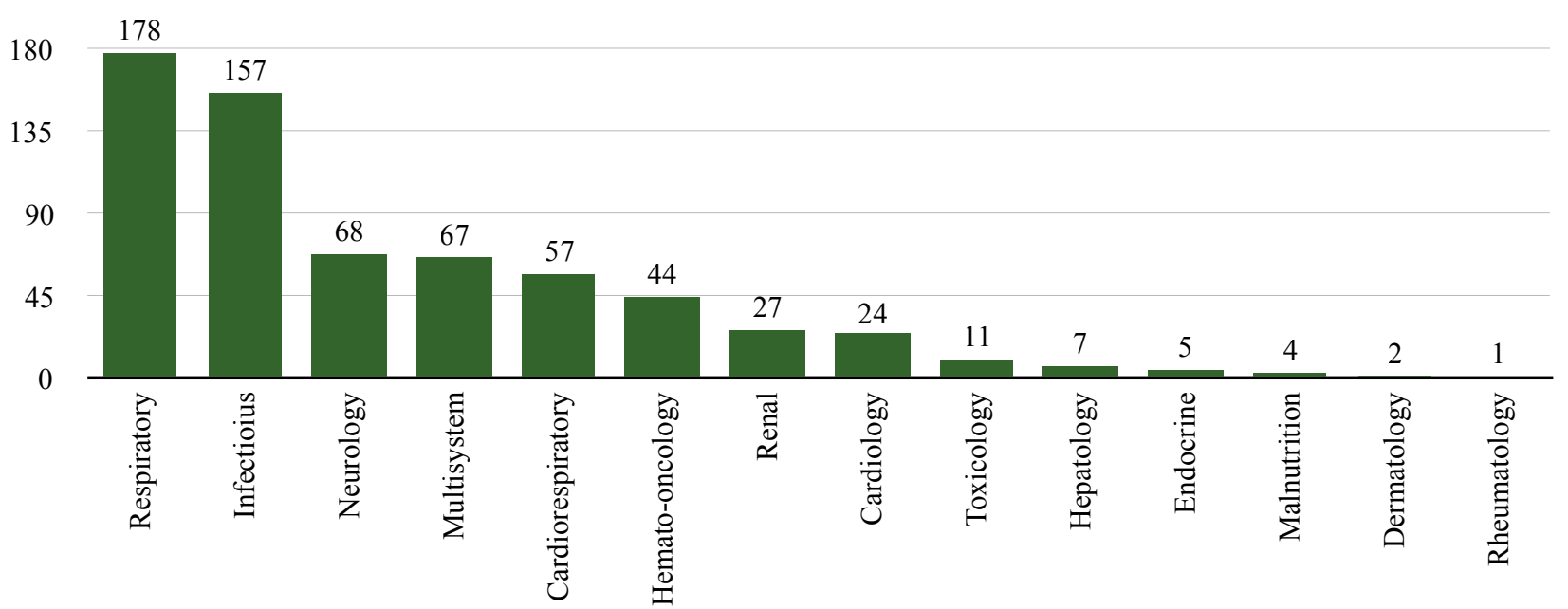

Figure 1. System-wise distribution of patients

encephalopathy. Other less common reasons were as in Figure 2. Miscellaneous group includes GBS, DKA, severe dehydration, DIC, ALF, hypertensive emergency and tetanic spasms.

Analysis of outcome showed that out of 652 patients, 484 patients improved. Two $(0.3 \%)$ cases were referred to other centre, $46(7.05 \%)$ cases left against medical advice (LAMA) and 120 (18.46\%) cases expired. Among 484 improved cases 479 $(73.46 \%)$ were transferred out to the respective ward/cabin and five $(0.76 \%)$ were discharged directly from PICU in satisfactory condition.

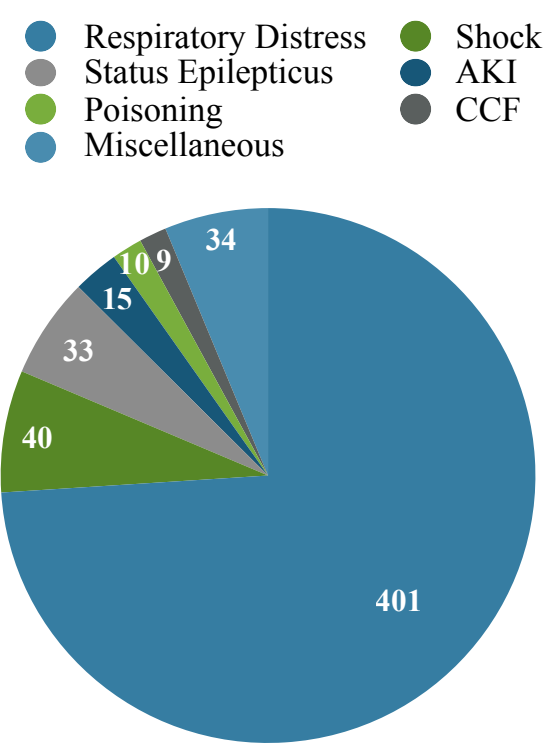

Figure 2. Reason for PICU admission
For the purpose of analysing final out-come, patients were categorised as survivors and non survivors. Survivors $(\mathrm{n}=484,74.23 \%)$ included either transferred to ward or discharged directly from PICU. Those who expired and whose final status remained unknown such as LAMA and referred were considered as non-survivors ( $\mathrm{n}=168$; $25.76 \%$ ). Final outcome (survivor vs non-survivor) of the patients admitted in PICU is as shown in Table 3.

Total number of deaths was 120 with mortality rate being $18.4 \%$. Mortality among, infants less than one year, one to five years and above five years of age were $47.5 \%(\mathrm{n}=57), 21.66 \%(\mathrm{n}=26)$ and $30.83 \%(\mathrm{n}=37)$ respectively. Mortality among infant less than one year was significantly high as compared to other age group $[\mathrm{p}$ value $=0.03, \mathrm{OR}$ $1.45(1.02-2.07)]$. Median age was 12 months. Out of 120 patients who died, $70(58.33 \%)$ were males as compared to $50(41.66 \%)$ females. Approximately one third of the deaths $(n=44$; $36.7 \%$ ) occurred within 48 hours of admission in PICU $(p<0.05)$. Case fatality was highest for acute leukemia $(37.5 \%)$ and Other Infectious diseases group (30\%) followed by AES (26.1\%) and sepsis $(23.6 \%)$.

Length of stay (LOS) in the PICU ranged from a few hours to 47 days, with a mean of $5.72 \pm 5.78$ days and median of four days. For analytic purpose LOS was categorised in three groups, those staying for less than 48 hours, three to seven days and more than seven days. Around 188 (28.8\%) cases stayed 
Table 3. Final Outcome of patients admitted in the PICU

\begin{tabular}{|c|c|c|c|c|c|}
\hline \multirow{2}{*}{ Variables/Diseases } & \multirow{2}{*}{$\begin{array}{l}\text { Admission } \\
\text { No. }(\%)\end{array}$} & \multicolumn{2}{|c|}{ Final Outcome No. (\%) } & \multirow[t]{2}{*}{ p Value } & \multirow[t]{2}{*}{ Odds Ratio } \\
\hline & & Survivors & Non-survivors & & \\
\hline \multicolumn{6}{|l|}{ Age } \\
\hline$<12$ months & $352(53.98)$ & $273(77.6)$ & $79(22.4)$ & \multirow{3}{*}{0.07} & \\
\hline $1-5$ years & $132(20.24)$ & $96(72.7)$ & $36(27.3)$ & & \\
\hline$>5$ years & $168(25.76)$ & $115(68.5)$ & $53(31.5)$ & & \\
\hline \multicolumn{6}{|l|}{ Gender } \\
\hline Male & $397(60.88)$ & $298(75.06)$ & $99(24.93)$ & \multirow[t]{2}{*}{0.54} & \multirow{2}{*}{$0.89(0.62-1.28)$} \\
\hline Female & $255(39.11)$ & $186(72.94)$ & $69(27.05)$ & & \\
\hline \multicolumn{6}{|l|}{ Length of Stay } \\
\hline$<48$ hours & $188(28.83)$ & $125(66.48)$ & $63(33.52)$ & \multirow{3}{*}{$<0.001$} & \\
\hline $3-7$ days & $321(49.2)$ & $264(82.24)$ & $57(17.76)$ & & \\
\hline$>7$ days & $143(21.9)$ & $95(66.43)$ & $48(33.57)$ & & \\
\hline \multicolumn{6}{|l|}{ Diseases } \\
\hline Pneumonia & 216 & $168(77.77)$ & $48(22.22)$ & 0.14 & $1.33(0.91-1.95)$ \\
\hline Sepsis & 123 & $81(65.85)$ & $42(34.15)$ & 0.01 & $0.60(0.39-0.92)$ \\
\hline Bronchiolitis & 44 & $43(97.7)$ & $1(2.3)$ & 0.00 & $16.28(2.22-119.1)$ \\
\hline Other infectious diseases & 38 & $23(60.5)$ & $15(39.5)$ & 0.04 & $0.50(0.25-1.0)$ \\
\hline AES & 23 & $14(60.87)$ & $9(39.13)$ & 0.13 & $0.52(0.22-1.23)$ \\
\hline Seizure disorder & 24 & $20(83.3)$ & $4(16.7)$ & 0.29 & $1.76(0.59-5.25)$ \\
\hline $\begin{array}{l}\text { AGE with severe } \\
\text { dehydration }\end{array}$ & 19 & $16(84.2)$ & $3(15.8)$ & 0.31 & $1.88(0.54-6.53)$ \\
\hline Meningitis & 18 & $12(66.7)$ & $6(33.33)$ & 0.45 & $0.68(0.25-1.85)$ \\
\hline Congenital heart diseases & 17 & $12(70.6)$ & $5(29.4)$ & 0.72 & $0.83(0.29-2.38)$ \\
\hline Acute leukemia & 16 & $6(37.5)$ & $10(62.5)$ & 0.001 & $0.19(0.07-0.55)$ \\
\hline Other oncological disorders & 14 & $9(64.3)$ & $5(35.7)$ & 0.39 & $0.62(0.20-1.87)$ \\
\hline Nephrotic syndrome & 12 & $11(91.7)$ & $1(8.3)$ & 0.16 & $3.8(0.49-30.31)$ \\
\hline Poisoning & 11 & $11(100)$ & - & 0.04 & $1.35(1.29-1.41)$ \\
\hline Co-morbidities & $141(21.62)$ & $88(62.4)$ & $53(37.6)$ & 0.001 & $2.07(1.39-3.09)$ \\
\hline
\end{tabular}

for less than 48 hours, whereas $321(49.2 \%)$ cases remained in PICU between three to seven days and $143(21.9 \%)$ cases for more than seven days. Average LOS for survivors vs non-survivors was $5.54 \pm 5.26$ days and $6.22 \pm 7.06$ days respectively ( $\mathrm{p}$ value $=0.00)$. Bed occupancy was maximum and minimum in the months of May 74 (11.5\%) and June 28 (4.3\%) respectively. Maximum number of patients were from Province three (Bagmati) accounting for $313(48 \%)$ cases. Other geographical distribution has been depicted below in Fig 3. 

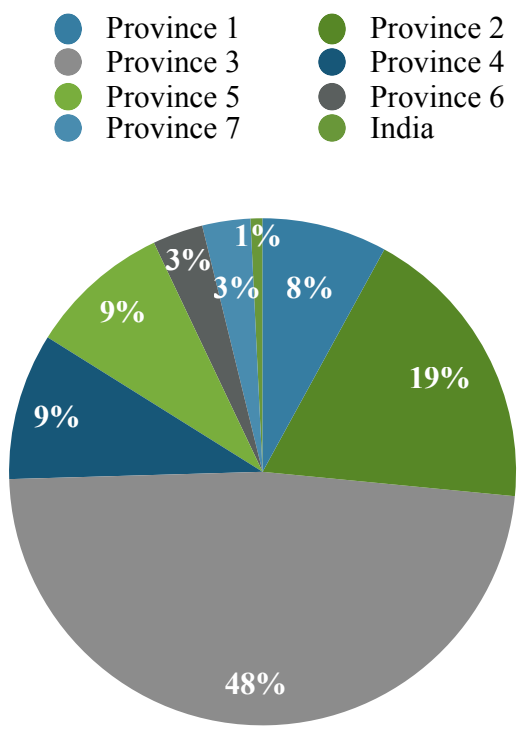

Figure 3. Geographical distribution of patients

\section{DISCUSSION}

Caring for critically ill children is a challenge in developing countries, where necessary equipment is scarce and trained manpower is limited. ${ }^{10}$ Due to the scarcity of published data of PICU profile within Nepal, adequate comparison of relative incidence and outcome is not possible within the country.

In this study, 652 cases were admitted with an average of 54.3 cases / month and bed occupancy rate of $85.18 \%$, which was much higher than that was seen in Patan Academy of Health Sciences (PAHS) $(\mathrm{n}=126)$ and B.P Koirala Institute of Health Sciences (BPKIHS) $(n=230))^{4,7}$ This was probably because ours is the only Government paediatric hospital where majority of patients from low socioeconomic status come. Similar impression was made by Khanal A et al. in the year 2016. ${ }^{3}$

Age on admission ranged from 1 month to 14 years with a mean of $34.18 \pm 45.48$ months and median of nine months. Maximum number of patients $(\mathrm{n}=$ $352,53.98 \%$ ) were infants in the age group of one month to one year as seen in BPKIHS (31.3\%.), India (47.4\%) and Pakistan (42\%), however it is in contrast to the finding in Ethiopia where above five years of age contributed more than half $(57.8 \%)$ of the admission. ${ }^{1,2,7,11}$ The difference could be due to the difference in the pattern of diseases they encounter in their geographical set-up. Male babies out numbered their female counterpart $(60.88 \%$ vs $39.12 \%$ ) in our study which is in accordance with similar earlier studies at BPKIHS and PAHS where male patients accounted for $63 \%$ and $57 \%$ respectively. ${ }^{4,7}$ Similar insignificant male preponderance was found in other studies elsewhere possibly reflecting the social bias still prevailing against the girl child in Southeast Asia. ${ }^{1,2,10}$

We observed a wide spectrum of cases covering almost all the systems like respiratory $(\mathrm{n}=178$, $27.3 \%$ ), infections ( $\mathrm{n}=157,24.1 \%)$, neurology ( $=68,10.4 \%)$, cardiorespiratory $(\mathrm{n}=57,8.7 \%)$ Cardiac $(n=24,3.7 \%)$ and hemato-oncology $(n=$ 44, 6.7\%). Pneumonia (33.01\%) was the major disease requiring PICU admission as was reported in other earlier studies. ${ }^{5,710}$ This may be attributed to the fact that pneumonia is one of the leading causes of paediatric morbidity and mortality in South East Asia. ${ }^{12}$ In our study 161 children (24.69\%) were admitted for infectious diseases, significant number of which were due to sepsis $(\mathrm{n}=$ $123,18.9 \%$ ). This may be due to the fact that infectious diseases still contribute to a vast majority of illnesses in our part of the world. ${ }^{13}$ In contrast to our finding, GBS, RHD and post infectious glomerulonephritis were more prevalent in Ethiopia. ${ }^{11}$ This shows that paediatric intensive care admissions vary among different regions in different settings and one should be aware of the prevalent conditions to develop the facilities and prepare relevant treatment plan.

The study revealed that around $1.9 \%(n=13)$ of the patients discharged to the paediatric ward were readmitted to the PICU. Early discharge of less severe PICU patients to provide PICU beds for more severe critically ill patients can be a reasonable explanation for this. Establishment of a paediatric intermediate care unit as a step down unit will not only offer better care to such patients but also avail more vacant beds in the PICU.

Respiratory distress $(61.5 \%)$ was the major reason for PICU admission in our study, which is in accordance to the study done by Khanal $\mathrm{A}$ et al. revealing that $60 \%$ of PICU units within the 
country reported respiratory distress / failure as their primary cause for admission. ${ }^{3}$ But in PAHS Basnet $\mathrm{S}$ et al. reported shock (45\%), particularly septic shock (30\%) as the main reason for PICU admission which was the second common cause in our study. ${ }^{4}$ The discrepancy in these findings may be just because of the differences in the morbidity patterns of the study population. Neurological causes like status epilepticus and poor GCS were other common reasons requiring PICU admission in our study as has been reported in earlier study. 3,4

Among the total study population, 484 (74.23\%) patients improved and were transferred to ward or discharged to home. Several previous studies showed nearly similar pattern of improvement ranging between $68 \%$ to $77 \%$. $^{7,10,14}$ Two $(0.3 \%)$ cases were referred to other centre for not having cardio-thoracic and neurosurgical facility in our centre and 46 (7.05\%) cases went on LAMA. Most of these patients left the hospital either due to financial constraints or parents' perception that the child won't survive from current illness which is in accordance with various other published studies. ${ }^{7,15}$

Mortality rate in our study was $18.5 \%$. This rate may be considered acceptable, as mortality rates between $12.6 \%$ - 19\% have been reported from PICUs in other parts of the world. 2,7,10,14 However, a higher mortality rate was reported in another study within the country. ${ }^{4}$ This could be attributed to the fact that there is an inverse relationship between patient volume and outcomes in the setting of the PICU. PICUs with greater volumes had reductions in both PICU mortality risk and PICU length of stay. ${ }^{16}$ Factors contributing to high morbidity and mortality in ICUs are poor healthcare system, length of illness, delayed presentation, history of treatment before PICU admission, level of clinical instability while being treated and severity of disease when the patients presented to the hospital. ${ }^{14,17}$

Though age related mortality in our PICU was highest $(47.5 \%)$ for the age group of less than one year and more male patients died as compared to female ( 74 vs 46 ), age and sex did not show any statistically significant difference between survivor and non-survivor. Contrary to our findings, patients within the age group of one to five years were having highest mortality in AIIMS (32.1\%) and BPKIHS (7.8\%). 5,7 This could be because infants were the major contributor for PICU admission in our hospital.

Case fatality was highest for acute leukemia $[37.5 \% ; \mathrm{p}=0.04]$ and other infectious diseases group $[30 \% ; p=0.08]$, whereas study in Bangladesh showed septicemia (33.3\%) and pneumonia $(24 \%)$ as top fatal diseases. ${ }^{10}$ Combination of increased susceptibility to infections, inadequate nutrition, cachexic state and bone marrow suppression caused by the use of chemotherapeutic agents might be the reason behind high fatality among oncological cases. The mortality and morbidity of infectious diseases and sepsis remain high despite widespread advances in treatment. ${ }^{18}$

Certain diseases among admitted patients were of statistical significance when compared between survivors and non-survivors. Bronchiolitis (97.7\% vs $2.3 \%)$, sepsis $(65.85 \%$ vs $34.15 \%)$, other infectious diseases $(60.5 \%$ vs $39.5 \%)$ and poisoning ( $100 \%$ vs nil) had the best prognosis, while acute leukemia $(37.5 \%$ vs $62.5 \%)$ had the worst prognosis.

In our study around $21.77 \%(\mathrm{n}=142)$ of patients had co-morbidities and most of these were cardiac and neurological. We found that survival in patients without comorbidity $(77.5 \%)$ was two times higher than in patients with co-morbidities $(62.4 \%)$ [p $<0.001$; OR $2.074(1.392-3.091)]$. This pattern of association of co-morbidities was previously reported in Greece. ${ }^{6}$

The average LOS in our unit was $5.72 \pm 5.78$ days and median of four days regardless of outcome whereas mean of $6.2 \pm 5.3$ days and median of four days was seen at PAHS. ${ }^{4}$ As in Egypt (78.4\%) majority of the children in our study $(78.06 \%)$ remained in PICU for less than seven days. ${ }^{14} \mathrm{We}$ found the average LOS for survivor and nonsurvivor was $5.54 \pm 5.26$ and $6.22 \pm 7.06$ days respectively. The LOS was significantly shorter for survivor $(5.54 \pm 5.26$ vs $6.22 \pm 7.06$ days; $p<$ 0.05 ) as compared to non-survivor but maximum death $(36.7 \%)$ occurred within 48 hours of admission. This controversy could be because some 
ICU admissions were for patients who had grim chances of recovery and succumbed to death early. Large number of LAMA cases in later group could have prolonged the stay of the same. Similar pattern of shorter stay for survivor as compared to non-survivors $(8.50 \pm 22.11$ vs $12.17 \pm 32.58$ days $)$ was reported from Greece. ${ }^{19}$

Our study incorporated a variety of patients with different illnesses reflecting the wider service facility of central PICU. Despite this and large sample size, our study was limited by the fact that it was a single centric study, the result of which may not be generalised to the population of various areas where resources are more limited. Also, being a retrospective study, data collected were incomplete in terms of use of mechanical ventilation which couldn't be analysed. A wider study needs to be conducted that encompasses the outcome of mechanical ventilations as well.

\section{CONCLUSIONS}

Pneumonia with respiratory distress was the major cause of admission in PICU, followed by sepsis, bronchiolitis and other infectious diseases. Case fatality was highest for acute leukemia. Almost one fifth of the patients had some form of comorbidity. The results of this study confirm that the mortality rate was positively correlated with association of co-morbidities, LOS and younger age.

\section{REFERENCES}

1. Sahoo B, Patnaik S, Mishra R, Jain MK. Morbidity Pattern and Outcome of Children Admitted to a Paediatric Intensive Care Unit of Eastern India. Int J Contemp Pediatr. 2017;4(2):486-9. DOI: 10.18203/2349-3291.ijcp20170694

2. Khursid A, Khan MA, Saleem M, Zafar F. Clinical Profile and Outcome in a Paediatric Intensive Care Unit in Pakistan. Pak J Med Health Sci. 2015;9(2):623-5.

3. Khanal A, Sharma A, Basnet S. Current State of Paediatric Intensive Care and High Dependency Care in Nepal. PCCM Journal. 2016;17(11):1032-1040. DOI: 10.1097/PCC.0000000000000938

4. Basnet S, Shrestha S, Ghimire A. Development of a PICU in Nepal: The Experience of the First Year. PCCM Journal. 2014 September; 15 (7):e314-e320.DOI: 10.1097/PCC.0000000000000201

5. Khilnani P, Sarma D, Singh R. Demographic Profile and Outcome Analysis of a Tertiary Level Pediatric Intensive Care Unit. Apollo Medicine. 2004 December;1 (2):161-166. DOI: 10.1016/S0976-0016(11)60242-1

6. Briassoulis G, Filippou O, Natsi L, Mavrikiou Ma, Hatzis T. Acute and Chronic Paediatric Intensive Care Patients: Current Trends and Perspectives on Resource Utilization. Q J Med. 2004;97(8):507-518. DOI:10.1093/qjmed/ hch087

7. Shah GS, Shah BK, Thapa A, Shah LR, Mishra OP. Admission Patterns and Outcome in a Paediatric Intensive Care Unit in Nepal. Brit J Med and Med Resear. 2014 October;4 (s30):4939-4945. DOI: https://doi.org/10.9734/ BJMMR/2014/10318

8. Ministry of Health and Population GoN. HMIS Indicators. In: Services DoH, ed. 1st ed. Teku,Kathmandu,Nepal: Health Management Information Section; 2070:20.

9. Sindhu J, Reddy K, Satyanarayana N, Devaraya S, Fathima A. Hospital Utilisation Statistics: Thirty-Five Year Trend Analysis, A Measure of Operational Efficiency of a Tertiary Care Teaching Institute in South India. IOSR Journal of Dental and Medical Sciences. 20191;18(4):49-55. DOI: 10.9790/0853-1804024955

10. Hoque M, Masud M, Ahmed A. Admission Pattern and Outcome in a Paediatric Intensive Care unit of a Tertiary Care Paediatric Hospital in Bangladesh - A two-year analysis. DS (Child) H J. 2012;28(1):14-9.

11. Tazebew A, Cahkilu B, Bacha T. Admission Pattern and Outcome In A Paediatric Intensive Care Unit of Gondar University Hospital. Ethiop Med J. 2019;57(2). 
12. Ghimire M, Bhattacharya S, Narain J. Pneumonia in South-East Asia Region: Public Health Perspective. Indian J Med Res. 2012;135(4):459-68. PMID: 22664492.

13. Adhikari N, Avila ML, Kache S, Grover T, Ansari I, Basnet S. Establishment of Paediatric and Neonatal Intensive Care Units at Patan Hospital, Kathmandu: Critical Determinants and Future Challenges. J Nepal Paediatr Soc. 2011;31(1):49-56. DOI: https://doi.org/10.3126/jnps.v31i1.4161

14. Abdallah NB, Zeitoun AE, Abdel Fattah MGE. Adherence to Standard Admission and Discharge and its Association With Outcome of Pediatric Intensive Care Unit in Al-Ahrar Hospital Zagazig. Egyptian Pediatric Association Gazette. 2016;64:111-9. DOI: 10.1016/j.epag.2016.08.002

15. Somasetia DH, Isman S, Wonoputri N. Discharge Against Medical Advice in Paediatric Intensive Care Unit and Subsequent Events. IJIHS. 2015;3(1):7-10. DOI: 10.15850/ijihs.v1n1.102

16. Tilford JM, Simpson PM, Green JW, Lensing Sa, Fiser DH. Volume-Outcome Relationships in Paediatric Intensive Care Units. Pediatrics. 2000;106(2):289-94. DOI: 10.1542/peds.106.2.289

17. Dünser MW, Baelani I, Ganbold L. A Review and Analysis of Intensive Care Medicine in The Least Developed C34ountries. Crit Care Med. 2006(4):1234-42. DOI: 10.1097/01.CCM.0000208360.70835.87

18. Nawawy AE. Evaluation of the Outcome of Patients Admitted to the Paediatric Intensive Care Unit in Alexandria Using the Pediatric Risk of Mortality (PRISM) Score. Journal of Tropical Paediatrics. 2003;49(2):109-114. DOI: 10.1093/tropej/49.2.109

19. Volakli E, Sdougka M, Tamiolaki M, Tsonidis C, Reizoglou M, Giala M. Demographic Profile and Outcome Analysis of Paediatric Intensive Care Patients. Hippokratia. 2011;15(4):316-322. PMID: 24391412 